\title{
AVALIAÇÃO DA INFRAESTRUTURA DAS UNIDADES DE SAÚDE DA FAMÍLIA E EQUIPAMENTOS PARA AÇÕES NA ATENÇÃO BÁSICA
}

\author{
Kênia Souto Moreira ${ }^{1}$, Cássio de Almeida Lima², Maria Aparecida Vieira ${ }^{3}$, Simone de Melo Costa ${ }^{4}$
}

\begin{abstract}
RESUMO: Objetivou-se avaliar a infraestrutura das unidades de saúde da família e os equipamentos para ações na Atenção Básica. Pesquisa quantitativa, transversal analítica, realizada em 2014 em cidade polo de Minas Gerais, com avaliação de subdimensão da Autoavaliação para Melhoria do Acesso e da Qualidade da Atenção Básica, de 75 equipes. A classificação da subdimensão situou-se no padrão insatisfatório para $48 \%$ das unidades. São poucas as unidades com total adequação para: ações em saúde $(5,3 \%)$, atendimento com privacidade $(9,3 \%)$, disposição de telefone e internet (1,3\%), recursos para atender urgência e emergência (1,3\%), veículo oficial para ações externas (4\%), identificação visual das dependências e dos profissionais (4\%). A disposição de telefone e internet apresentou maior média para zona urbana e disposição de veículo oficial foi maior para zona rural $(p<0,05)$. A qualidade da infraestrutura e dos equipamentos obteve avaliação predominantemente negativa pelas equipes, limitando a consolidação da Atenção Básica.
\end{abstract}

DESCRITORES: Serviços de saúde; Avaliação de serviços de saúde; Qualidade da assistência à saúde; Estratégia saúde da família; Atenção primária à saúde.

\section{ASSESSMENT OF INFRASTRUCTURE OF FAMILY HEALTH UNITS AND EQUIPMENT USED IN PRIMARY CARE ACTIONS}

ABSTRACT: The present study aimed to assess the quality of infrastructure in family health units and of the equipment used in primary care actions. Quantitative, cross-sectional analytical study conducted in 2014 in a large city of Minas Gerais, with assessment of the subdimension Self-assessment for Access and Quality Improvement in Primary Care of 75 teams. The subdimension was considered unsatisfactory by $48 \%$ of the units. Few units met all the requirements assessed for all health actions: $(5.3 \%)$ regarding the delivery of private, personalized health care services; (9.3\%) regarding availability of telephone line and internet connection; $(1.3 \%)$ regarding resources needed to meet emergency care needs; $(1.3 \%)$ regarding the availability of a vehicle for the transportation of the health teams in planned external activities; $(4 \%)$ regarding the visual identification of the facilities and teams. The availability of a telephone line and internet connection showed the highest average in urban areas and the availability of a vehicle for transportation of the teams was higher in rural areas $(p<0.05)$. The assessment of the quality of infrastructure and equipment by the teams was predominantly negative, impacting the consolidation of Primary Care.

DESCRIPTORS: Health services; Assessment of health services; Quality of health care; Family health strategy; Primary health care.

\section{EVALUACIÓN DE LA INFRAESTRUCTURA DE LAS UNIDADES DE SALUD DE LA FAMILIA Y DE LOS EQUIPOS PARA ACCIONES EN LA ATENCIÓN BÁSICA}

RESUMEN: Estudio cuyo objetivo fue evaluar la infraestructura de las unidades de salud de la família, así como los equipos para acciones en la Atención Básica. Investigación cuantitativa, transversal analítica, realizada en 2014 en ciudad polo de Minas Gerais, por medio de evaluación de subdimensión de la Autoevaluación para Mejorar el Acceso y la Cualidad de la Atención Básica de 75 equipos. Se evaluó la clasificación de la subdimensión como insatisfactoria para $48 \%$ de las unidades. Fueron pocas unidades con total adecuación para: acciones en salud (5,3\%), atendimiento con privacidad $(9,3 \%)$, disposición de teléfono e internet $(1,3 \%)$, recursos para atender urgencias y emergencias (1,3\%), vehículo oficial para acciones externas $(4 \%)$, identificación visual de las dependencias y de los profesionales (4\%). La disposición de teléfono e internet presentó media mayor para zona urbana y hubo más disposición de vehículo oficial en el ámbito rural $(p<0,05)$. La evaluación de la cualidad de la infraestructura y de los equipos por los profesionales fue predominantemente negativa, limitándose la consolidación de la Atención Básica.

DESCRIPTORES: Servicios de salud; Evaluación de servicios de salud; Cualidad de la asistencia a la salud; Estrategia Salud de la familia; Atención primaria a la salud.

${ }^{1}$ Enfermeira. Mestre em Cuidado Primário em Saúde. Docente das Faculdades Integradas Pitágoras de Montes Claros. Montes Claros, MG, Brasil.

${ }^{2}$ Enfermeiro. Mestrando em Saúde, Sociedade e Ambiente. Universidade Federal dos Vales do Jequitinhonha e Mucuri. Diamantina, MG, Brasil.

${ }^{3}$ Enfermeira. Doutora em Ciências. Docente de Enfermagem da Universidade Estadual de Montes Claros. Montes Claros, MG, Brasil.

${ }^{4}$ Cirurgiã-Dentista. Doutora em Odontologia-Saúde Coletiva. Docente da Universidade Estadual de Montes Claros. Montes Claros, MG, Brasil.

Autor Correspondente:

Recebido: $17 / 03 / 2017$

Cássio de Almeida Lima

Finalizado: 22/05/2017

Universidade Federal dos Vales do Jequitinhonha e Mucuri

Rodovia MGT 367 - Km 583, 5000 - 39100-000 - Diamantina, MG, Brasil

E-mail: cassioenf2014@gmail.com 


\section{INTRODUÇÃO}

A expansão da Estratégia Saúde da Família (ESF) coloca em discussão questões sobre qualificação das equipes de saúde, resolubilidade dos serviços e necessidade de monitorar e avaliar a Atenção Básica (AB). Para isso, o Ministério da Saúde brasileiro passa a defender o aprimoramento contínuo de processos avaliativos na rotina da gestão e dos serviços de saúde pública. A Política de Monitoramento e Avaliação da $A B$ instituiu a Autoavaliação para Melhoria do Acesso e da Qualidade da Atenção Básica (AMAQ), em conjunto com estados e municípios, estabelecendo a avaliação como instrumento permanente para a tomada de decisões e a qualidade como um atributo fundamental a ser alcançado no Sistema Único de Saúde (SUS) ${ }^{(1-4)}$.

Nesse contexto, a AMAQ analisa, entre outras dimensões, a infraestrutura e os equipamentos indispensáveis para o desenvolvimento das ações na AB. Ressalta-se que a estrutura física adequada e a disponibilidade de equipamentos e materiais, com os recursos necessários para a execução de ações em saúde e em correspondência quantitativa ao contingente populacional adscrito e suas especificidades, é de fundamental importância para organização dos processos de trabalho e atenção à saúde dos usuários, a fim de se oferecer uma assistência de melhor qualidade(1).

No entanto, na prática em saúde da família evidencia-se uma necessidade de aprimoramento das características físico-estruturais e de obtenção de equipamentos e materiais requeridos para a realização das práticas em saúde. As deficiências estruturais das unidades repercutem em insatisfação dos profissionais das equipes de saúde da família. Isso porque o modelo de assistência proposto na ESF se coloca a favor de uma assistência integral ao indivíduo, à família e à comunidade, o que requer condições estruturais mínimas necessárias para a execução das ações que ultrapassam o modelo biomédico ${ }^{(5-6)}$.

Sendo assim, a estrutura deve ser valorizada na avaliação dos serviços de Atenção Primária à Saúde. A avaliação da infraestrutura e dos equipamentos constitui instrumento importante na prática gerencial ${ }^{(7)}$. Deve ser estimulada e seus conhecimentos aplicados e discutidos no sentido de aumentar o desempenho e o impacto das ações na $\mathrm{AB}$ junto à saúde da população ${ }^{(4,8-9)}$. Desse modo, os estudos poderão subsidiar o aprofundamento do conhecimento da realidade específica das unidades de saúde para o planejamento de intervenções que possam melho $\neg$ rar as condições de atendimento ${ }^{(5,10)}$. Torna-se importante aferir a autoavaliação das equipes sobre suas unidades de saúde por meio do instrumento da AMAQ, o qual permite identificar as subdimensões que exigem maior atenção e intervenção da gestão $^{(2-4)}$.

Este estudo teve como objetivo avaliar a infraestrutura das unidades de saúde da família e os equipamentos para ações na Atenção Básica.

\section{MÉTODO}

Trata-se de estudo com abordagem quantitativa, desenho transversal e analítico. Teve como cenário a cidade polo do Norte do estado de Minas Gerais, Montes Claros. Conforme censo demográfico do Instituto Brasileiro de Geografia e Estatística, o contingente populacional do município foi estimado em 394.350 mil habitantes ${ }^{(11)}$.

O estudo foi conduzido com dados de todas as 75 equipes de saúde da família, da zona urbana e rural, cadastradas no município. Os dados foram coletados em 2014, a partir do instrumento AMAQ, em site do Ministério da Saúde, sendo os arquivos impressos e disponibilizados por técnicos da secretaria municipal de saúde.

AMAQ é um instrumento referente à segunda fase de desenvolvimento do Programa de Monitoramento e Avaliação de Qualidade (PMAQ). É uma ferramenta de avaliação de serviços de saúde gerada a partir de instrumentos validados, nacionalmente e internacionalmente, e foi utilizada pelo Ministério da Saúde brasileiro. O instrumento é composto por duas dimensões e subdimensões relacionadas à equipe de atenção básica, com análise independente. Neste estudo, analisou-se a subdimensão Infraestrutura e Equipamentos, integrante da dimensão Unidade Básica de Saúde (UBS) 
A subdimensão avaliada considerou oito padrões de qualidade da UBS:

- A UBS, considerando sua infraestrutura física e equipamentos, está adequada para o desenvolvimento das ações;

- A UBS dispõe de consultórios com infraestrutura e equipamentos básicos que permitem o atendimento individual dos usuários com garantia de privacidade visual e auditiva;

- A UBS possui cronograma de manutenção das instalações físicas, equipamentos e instrumentais de forma regular e sistemática;

- A UBS dispõe de linha telefônica, equipamentos de informática com acesso à internet para os profissionais desempenharem suas atividades;

- A UBS dispõe dos materiais e equipamentos necessários ao primeiro atendimento nos casos de urgência e emergência;

- O deslocamento dos profissionais das equipes, para a realização de atividades externas programadas, quando necessário, é realizado por meio de veículo oficial;

- A UBS está adequada para atendimento de pessoas com deficiência e/ou com mobilidade reduzida, analfabetos e idosos;

- A UBS possui identificação visual externa e interna em todas as suas dependências e dos profissionais ${ }^{(1)}$.

Cada item permite avaliar o grau de adequação da infraestrutura das unidades de saúde e os equipamentos indispensáveis para as ações na $A B$ ao padrão de qualidade apresentado, em escala que varia de zero a 10 pontos, sendo zero o não cumprimento ao padrão e 10 a total adequação. Os pontos são somados para classificar a equipe em resultados muito insatisfatório, insatisfatório, regular, satisfatório e muito satisfatório, conforme zero-15 pontos, 16-31, 32-47, 48-63 e 64-80, respectivamente. Quanto maior a pontuação, melhor a classificação da equipe ${ }^{(1)}$. Além das oito questões do AMAQ, coletou-se informação acerca das equipes de saúde da família quanto à localização: urbana ou rural.

A análise estatística foi realizada por meio do software IBM Statistical Package for the Social Sciences (SPSS) Statistics, versão 22.0. A descrição dos resultados foi apresentada em valores percentuais, médias, desvio padrão, moda e percentis. Realizaram-se análises bivariadas para comparar as proporções de equipes em cada padrão de qualidade (muito insatisfatório a muito satisfatório) conforme localização (urbana, rural) pelo teste Likelihood ratio, alternativo do qui-quadrado de Pearson. Verificou-se a normalidade da distribuição dos dados pelo teste Kolmogorov Smirnov. Todas as oito questões de avaliação de qualidade apresentaram $\mathrm{p}<0,05$, ou seja, dados não paramétricos. $E$, para a questão do somatório dos pontos na subdimensão obteve-se o valor $p=0,464$. Sendo assim, utilizou-se o teste Mannn Whitney para comparar as médias das questões e o teste $t$ student para comparar as médias do somatório de pontos conforme localização rural ou urbana. Todos os testes consideraram o nível de significância $\mathrm{p}<0,05$.

A pesquisa foi conduzida em conformidade com as normas regulamentadoras das pesquisas envolvendo seres humanos. O projeto de pesquisa foi aprovado pelo Comitê de Ética em Pesquisa da Universidade Estadual de Montes Claros, Parecer Consubstanciado $n^{\circ}$ 704.718/2014. O projeto também obteve concordância institucional da Secretaria Municipal de Saúde.

\section{RESULTADOS}

Para as oito questões integrantes da subdimensão avaliada pelas equipes, observou-se que apenas quatro $(5,3 \%)$ equipes avaliaram a adequação das UBS com a pontuação 10; a falta de qualquer manutenção regular e sistemática foi destacada por 22 (29,3\%); assim como de telefone e internet, 14 $(18,7 \%)$; e a falta total de adequação da UBS para atender deficientes, analfabetos e idosos foi constatada para $15(20 \%)$ das equipes (Tabela 1$)$. 
Tabela 1 - Descrição dos pontos aos oito padrões de qualidade da subdimensão Infraestrutura e Equipamentos da Unidade Básica de Saúde. Equipes de saúde da família. Montes Claros, MG, Brasil, 2014

\begin{tabular}{|c|c|c|c|c|}
\hline Pontos & $\begin{array}{c}\text { UBS adequada para as } \\
\text { ações } \\
\text { n(\%) }\end{array}$ & $\begin{array}{c}\text { UBS permite } \\
\text { atendimento individual } \\
\text { com privacidade } \\
\mathbf{n}(\%)\end{array}$ & $\begin{array}{c}\text { UBS possui } \\
\text { manutenção regular e } \\
\text { sistemática } \\
\mathbf{n}(\%)\end{array}$ & $\begin{array}{l}\text { UBS dispõe de telefone } \\
\text { e internet para suas } \\
\text { ações } \\
\text { n(\%) }\end{array}$ \\
\hline 0 & $0(0)$ & $1(1,3)$ & $22(29,3)$ & $14(18,7)$ \\
\hline 1 & $3(4)$ & $1(1,3)$ & $9(12)$ & $1(1,3)$ \\
\hline 2 & 12(16) & 12(16) & $10(13,3)$ & $8(10,7)$ \\
\hline 3 & $6(8)$ & $2(2,7)$ & $9(12)$ & $9(12)$ \\
\hline 4 & $14(18,7)$ & $4(5,3)$ & $4(5,3)$ & $9(12)$ \\
\hline 5 & $10(13,3)$ & $9(12)$ & $8(10,7)$ & $10(13,3)$ \\
\hline 6 & $14(18,7)$ & $5(6,7)$ & $1(1,3)$ & $2(2,7)$ \\
\hline 7 & $4(5,3)$ & $10(13,3)$ & $6(8)$ & $10(13,3)$ \\
\hline 8 & $7(9,3)$ & 12(16) & $6(8)$ & $7(9,3)$ \\
\hline 9 & $1(1,3)$ & 12(16) & $0(0)$ & $4(5,3)$ \\
\hline 10 & $4(5,3)$ & $7(9,3)$ & $0(0)$ & $1(1,3)$ \\
\hline Pontos & $\begin{array}{c}\text { UBS dispõe de } \\
\text { recursos para primeiro } \\
\text { atendimento de } \\
\text { urgência e emergência } \\
\text { n(\%) }\end{array}$ & $\begin{array}{c}\text { UBS fornece } \\
\text { veículo oficial para } \\
\text { deslocamento dos } \\
\text { profissionais para } \\
\text { ações externas } \\
\text { n(\%) }\end{array}$ & $\begin{array}{c}\text { UBS adequada para } \\
\text { atender deficientes, } \\
\text { analfabetos e idosos } \\
\text { n(\%) }\end{array}$ & $\begin{array}{c}\text { UBS tem identificação } \\
\text { visual externa e interna } \\
\text { das dependências e } \\
\text { dos profissionais } \\
\text { n(\%) }\end{array}$ \\
\hline 0 & $29(38,7)$ & $59(78,7)$ & $15(20)$ & $3(4)$ \\
\hline 1 & $6(8)$ & $2(2,7)$ & $11(14,7)$ & $3(4)$ \\
\hline 2 & $9(12)$ & $1(1,3)$ & $10(13,3)$ & $4(5,3)$ \\
\hline 3 & $10(13,3)$ & $1(1,3)$ & $11(14,7)$ & $3(4)$ \\
\hline 4 & $6(8)$ & $0(0)$ & $8(10,7)$ & $3(4)$ \\
\hline 5 & $7(9,3)$ & $2(2,7)$ & $9(12)$ & $8(10,7)$ \\
\hline 6 & $3(4)$ & $2(2,7)$ & $2(2,7)$ & $8(10,7)$ \\
\hline 7 & $1(1,3)$ & $1(1,3)$ & $7(9,3)$ & $8(10,7)$ \\
\hline 8 & $3(4)$ & $2(2,7)$ & $2(2,7)$ & $24(32)$ \\
\hline 9 & $0(0)$ & $2(2,7)$ & $0(0)$ & $8(10,7)$ \\
\hline 10 & $1(1,3)$ & $3(4)$ & $0(0)$ & $3(4)$ \\
\hline
\end{tabular}

Fonte: Dados da autoavaliação para melhoria do acesso e da qualidade da atenção básica, 2014.

No somatório da pontuação na subdimensão, por equipe, observou-se neste estudo uma variação de quatro a 55 pontos, com média igual a 30,84 $( \pm 11,784)$ pontos por equipe. Entre as oito questões, a menor média entre as equipes foi para a questão deslocamento dos profissionais para realização de atividades externas programadas por meio de veículo oficial, 1,33 ( $\pm 2,974)$; enquanto a maior média foi para unidade dispõe de identificação externa e interna nas suas dependências, 6,27 $( \pm 2,632)$ (Tabela 2). 
Tabela 2 - Valores médios e separatrizes dos pontos obtidos nas oito questões de avaliação de Infraestrutura e Equipamentos na Unidade Básica de Saúde. Equipes de saúde da família. Montes Claros, MG, Brasil, 2014

\begin{tabular}{|c|c|c|c|c|c|c|}
\hline Questão & Média & $\begin{array}{l}\text { Desvio } \\
\text { padrão }\end{array}$ & Mínimo & Máximo & 25 & $\begin{array}{c}\text { Percentis } \\
50 \\
\text { mediana } \\
\end{array}$ \\
\hline UBS adequada para as ações & 4,91 & 2,332 & 1 & 10 & 3 & 5 \\
\hline $\begin{array}{l}\text { UBS permite atendimento individual com } \\
\text { privacidade }\end{array}$ & 6,21 & 2,777 & 0 & 10 & 4 & 7 \\
\hline UBS possui manutenção regular e sistemática & 2,77 & 2,679 & 0 & 8 & 0 & 2 \\
\hline UBS dispõe de telefone e internet para suas ações & 4,19 & 2,912 & 0 & 10 & 2 & 4 \\
\hline $\begin{array}{l}\text { UBS dispõe de recursos para primeiro } \\
\text { atendimento de urgência e emergência }\end{array}$ & 2,29 & 2,487 & 0 & 10 & 0 & 2 \\
\hline $\begin{array}{l}\text { UBS fornece veículo oficial para deslocamento } \\
\text { dos profissionais para ações externas }\end{array}$ & 1,33 & 2,947 & 0 & 10 & 0 & 0 \\
\hline $\begin{array}{l}\text { UBS adequada para atender deficientes, } \\
\text { analfabetos e idosos }\end{array}$ & 2,91 & 2,361 & 0 & 8 & 1 & 3 \\
\hline $\begin{array}{l}\text { UBS tem identificação visual externa e interna } \\
\text { das dependências e dos profissionais }\end{array}$ & 6,27 & 2,632 & 0 & 10 & 5 & 7 \\
\hline Soma total Infraestrutura e equipamentos & 30,84 & 11,784 & 4 & 55 & 23 & 30 \\
\hline
\end{tabular}

Fonte: Dados da autoavaliação para melhoria do acesso e da qualidade da atenção básica, 2014.

A classificação da subdimensão em padrões de qualidade foi insatisfatória para 36 (48\%) das UBS (Tabela 3).

Tabela 3 - Classificação da subdimensão Infraestrutura e Equipamentos da Unidade Básica de Saúde. Equipes de saúde da família. Montes Claros, MG, Brasil, 2014

\begin{tabular}{lcc} 
Infraestrutura e Equipamentos & & \\
\hline Classificação & $\mathbf{n}$ & $\mathbf{\%}$ \\
\hline Muito insatisfatório & 5 & 6,7 \\
\hline Insatisfatório & 36 & 48 \\
\hline Regular & 27 & 36 \\
\hline Satisfatório & 7 & 9,3 \\
\hline Total & $\mathbf{7 5}$ & $\mathbf{1 0 0}$
\end{tabular}

Fonte: Dados da autoavaliação para melhoria do acesso e da qualidade da atenção básica, 2014.

Não houve diferença significativa entre as médias de pontos obtidos na subdimensão avaliada conforme a localização das equipes, na zona rural a média foi 31,09 $( \pm 10,949)$ pontos e na zona urbana $30,80( \pm 12,003)$ pontos, $p=0,940$. Com relação às diferentes questões da subdimensão, conforme localização da UBS, constatou-se que a disposição de telefone e internet apresentou maior média para zona urbana, enquanto a disposição de veículo oficial para deslocamento dos profissionais para ações externas programadas foi maior para zona rural $(p<0,05)$, conforme descrito na Tabela 4. 
Tabela 4 - Comparação das médias de pontos nas questões de avaliação de Infraestrutura e Equipamentos na Unidade Básica de Saúde conforme localização urbana ou rural. Equipes de saúde da família. Montes Claros, MG, Brasil, 2014

\begin{tabular}{lccc} 
Questão & Urbana & Rural & p \\
\hline UBS adequada para as ações & 39,27 & 30,64 & 0,22 \\
\hline UBS permite atendimento individual com privacidade & 38,69 & 34 & 0,506 \\
\hline UBS possui manutenção regular e sistemática & 37,98 & 38,09 & 0,988 \\
\hline UBS dispõe de telefone e internet para suas ações & 40,63 & 22,73 & 0,011 \\
\hline $\begin{array}{l}\text { UBS dispõe de recursos para primeiro atendimento de urgência e } \\
\text { emergência }\end{array}$ & 37,43 & 41,32 & 0,572 \\
\hline $\begin{array}{l}\text { UBS fornece veículo oficial para deslocamento dos profissionais para } \\
\text { ações externas }\end{array}$ & 33,16 & 66,18 & $<0,001$ \\
\hline $\begin{array}{l}\text { UBS adequada para atender deficientes, analfabetos e idosos } \\
\text { UBS tem identificação visual externa e interna das dependências e dos } \\
\text { profissionais }\end{array}$ & 39,98 & 27,05 \\
\hline
\end{tabular}

Fonte: Dados da autoavaliação para melhoria do acesso e da qualidade da atenção básica, 2014.

Não houve associação entre classificação na subdimensão Infraestrutura e Equipamentos das Unidades Básicas de Saúde e localização urbana ou rural $(p=0,478)$. Tanto na zona urbana como na rural o maior percentual foi para UBS com padrão de qualidade insatisfatório, 29 (45,3\%) e sete (63,6\%), respectivamente (Tabela 5).

Tabela 5 - Classificação da subdimensão Infraestrutura e Equipamentos da Unidade Básica de Saúde conforme localização urbana ou rural. Equipes de saúde da família. Montes Claros, MG, Brasil, 2014

\begin{tabular}{lcccc} 
& Infraestrutura e Equipamentos da Unidade Básica de Saúde & \\
\cline { 1 - 4 } Classificação & $\begin{array}{c}\text { Zona urbana } \\
\mathbf{n}(\%)\end{array}$ & $\begin{array}{c}\text { Zona rural } \\
\mathbf{n}(\%)\end{array}$ & $\begin{array}{c}\text { Total } \\
\mathbf{n}(\%)\end{array}$ & $\begin{array}{c}\text { Likelihood Ratio } \\
\mathbf{p}\end{array}$ \\
\hline Muito Insatisfatório & $5(7,8)$ & $0(0)$ & $5(6,7)$ & 0,478 \\
\cline { 1 - 4 } Insatisfatório & $29(45,3)$ & $7(63,3)$ & $36(48)$ & \\
\hline Regular & $24(37,5)$ & $3(27,3)$ & $27(36)$ & \\
\cline { 1 - 4 } Satisfatório & $6(9,4)$ & $\mathbf{1}(9,1)$ & $7(9,3)$ & \\
\cline { 1 - 4 } Total & $\mathbf{6 4 ( 1 0 0 )}$ & $\mathbf{1 1 ( 1 0 0 )}$ & $\mathbf{7 5 ( 1 0 0 )}$ &
\end{tabular}

Fonte: Dados da autoavaliação para melhoria do acesso e da qualidade da atenção básica, 2014.

\section{DISCUSSÃO}

O presente estudo evidenciou uma avaliação majoritariamente negativa quanto aos equipamentos e à infraestrutura das unidades de saúde da família, por parte das equipes de saúde da família. Na literatura recente sobre a temática, as pesquisas apontam resultados similares aos observados neste trabalho ${ }^{(2-6,8,10,12-14)}$.

A infraestrutura frágil e a insuficiência de recursos materiais, além de com $\neg$ prometer $\mathrm{o}$ desenvolvimento e a qualidade das ações da $A B$, geram insatisfação nos profissionais e limitam as potencialidades de ampliação do elenco de ações na perspectiva da reorganização das práticas e do modelo de atenção à saúde ${ }^{(10)}$. Demonstrou-se na atual pesquisa que apenas $9,3 \%$ das equipes consideraram que a UBS está em total adequação para permitir o atendimento individual com 
privacidade. Em uma revisão sistemática, identificou-se que a falta de espaço físico adequado nas unidades tem levado à ausência de privacidade no diálogo com os usuários. Ademais, a carência de equipamentos e recursos materiais interfere na continuidade do atendimento e ocasiona condições de trabalho desfavoráveis ${ }^{(15)}$.

Em investigação de abrangência nacional, conduzida em 38.812 unidades básicas de saúde e 17.202 equipes de saúde participantes do PMAQ, com foco na adequação da estrutura na prevenção do câncer de colo de útero, a prevalência de adequação da estrutura foi $49 \%$. Tal prevalência foi maior entre as unidades cujo modelo assistencial era a ESF e entre aquelas que haviam aderido ao PMAQ $(61 \%)^{(16)}$. Outra pesquisa, realizada em 55 unidades da ESF de Cuiabá-MT, mostrou que a maioria das unidades contemplava quesitos de infraestrutura física e de oferta de ações no pós-parto ${ }^{(17)}$.

As falhas na infraestrutura e disponibilidade de insumos revelam ser necessário investir na melhoria da estrutura organizacional para que se agregue mais qualidade ao cuidado em saúde. Ressaltou-se em outro estudo que a disponibilidade de insumos e equipamentos na unidade de saúde foi o fator mais fortemente associado à melhor performance e ao desempenho mais adequado da $A B$, em Belo Horizonte- $\mathrm{MG}^{(18)}$.

Muitos serviços de saúde são instalados em prédios improvisados, que estão disponíveis nas comunidades e não possuem uma construção específica para atender aos usuários e aos profissionais de saúde, que esteja em conformidade com a legislação específica ${ }^{(14)}$. Grande parte das unidades básicas de saúde funciona em locais alugados, de modo que não se podem promover reformas ${ }^{(12,17)}$.

Ainda, foi preocupante a avaliação que os profissionais de saúde da família atribuíram à disponibilidade de linha telefônica e equipamentos de informática com acesso à internet, aos quais devem ter livre acesso. Achado ainda mais alarmante foi observado quanto à presença de veículo oficial para deslocamento para ações externas, sendo que 78,7\% dos profissionais indicaram pontuação zero para esse item. Similarmente, na avaliação da ESF em Campina Grande-PB, o trabalho burocrático não estava informati-zzado em nenhuma das unidades de saúde ${ }^{(8)}$. Essa deficiência anula as possibilidades de ganho advindo do uso das ferramentas eletrônicas destinadas ao armazenamento, organização, acesso e disponibilização da informação. E parte considerável do tempo dos profissionais acaba sendo ocupado, em detrimento de outras atividades, o que compromete a disponibilidade para a assistência ao usuário, impactando negativamente na qualidade do cuidado ${ }^{(8)}$.

O atual estudo constatou maior média de pontuação para disposição de linha telefônica e internet na zona urbana, e maior média de pontos para disposição de veículo oficial para deslocamento dos profissionais para ações externas programadas na zona rural. Esses resultados podem ser claramente elucidados pelo fato de que na área urbana há uma maior disponibilidade e acesso aos meios de comunicação e internet. Já, na zona rural, onde a organização territorial assume características específicas que demarcam maior distância entre as unidades de saúde e os domicílios, é uma condição mínima a presença de um automóvel para que as equipes possam realizar suas atividades.

Outro importante item avaliado na subdimensão infraestrutura e equipamentos é se a unidade de saúde dispõe de materiais e equipamentos necessários ao primeiro atendimento, nos casos de urgência e emergência. Nesse item, também predominou a avaliação negativa. Tem-se como hipóteses desse achado o baixo investimento destinado à aquisição de equipamentos para a $A B$, como também o caráter histórico dos atendimentos de urgência e emergência, comumente realizados apenas em hospitais e ambulatórios especializados. Em outro estudo conduzido na macrorregião Nordeste do estado de Minas Gerais ${ }^{(4)}$ apresentaram-se essas deficiências estruturais nas unidades básicas de saúde.

Sendo assim, é necessário intervir nesse aspecto, a fim de viabilizar o atendimento das equipes e, consequentemente, o aumento da resolubilidade da ESF(4). É válido ressaltar que, como a ESF constitui a porta de entrada do sistema de saúde, suas unidades devem estar equipadas para uma primeira abordagem nas situações de urgência e emergência. Ainda mais que, atualmente, o número de usuários portadores de doenças crônicas não transmissíveis tem aumentado consideravelmente, e esses pacientes poderão apresentar complicações, quadros de urgência e emergência, que irão requerer intervenções e ações iniciais no serviço da $A B$.

Neste estudo, verificou-se falta total de adequação das unidades de saúde para atender usuários portadores de deficiência, analfabetos e idosos para $20 \%$ das equipes. Resultado que merece atenção, 
pois os serviços de saúde da família devem garantir o amplo acesso e a acessibilidade da comunidade. Em Campina Grande ${ }^{(8)}$ e João Pessoa ${ }^{(14)}$, também constatou-se essa fragilidade. Como qualquer outro grupo populacional, as pessoas com deficiência necessitam de acesso à saúde. De acordo com a Política Nacional de Saúde da Pessoa com Deficiência, tanto a AB quanto os serviços de média e alta complexidade devem promover uma ampla cobertura no atendimento a esses usuários ${ }^{(14)}$.

Pesquisas internacionais também enfatizam a necessidade de melhorias na acessibilidade, pois a dificuldade ou impossibilidade de entrar em um estabelecimento é uma razão de pacientes com deficiência não procurarem os serviços de saúde ${ }^{(19-21)}$. Estudo realizado na Índia, com 839 pessoas com deficiência e 1153 sem deficiência, mostrou que aquelas com deficiência apresentaram uma necessidade de busca por serviços de saúde maior em comparação com as sem deficiência ${ }^{(20)}$.

Desse modo, em detrimento à impossibilidade do acesso ao serviço de saúde, esses usuários podem se tornar mais suscetíveis a doenças, como também retardar o tratamento quando já estão instaladas. Então, para se promover o acesso universal, é fundamental que as unidades de saúde disponham de adaptações ambientais adequadas ${ }^{(14)}$. Também devem dispor de materiais, equipamentos e instrumentos adequados para a promoção do acolhimento e da atenção humanizada e integral a pessoas com deficiência e/ou com mobilidade reduzida, analfabetos e idosos. Isso corresponde à ampliação do acesso, equidade e maior qualificação da atenção prestada ${ }^{(1)}$.

Face à avaliação das unidades de saúde da família apresentada neste estudo, observa-se que se trata de ter em mãos um sistema de saúde ideal, mas que necessita da operacionalização de suas formulações no cotidiano de suas ações ${ }^{(3)}$. Tal realidade suscita preocupações quanto à qualidade da expansão da ESF induzida nacionalmente. Uma provável explicação é que municípios de pequeno e médio porte encontram dificuldades no financiamento e na gestão local de seus sistemas de saúde, sendo necessária a consideração dos contextos específicos em que as políticas são implementadas ${ }^{(6)}$.

Todavia, mesmo que infraestrutura, equipamentos e insumos da ESF tenham sido foco de atenção, recentemente, por parte de governantes e gestores, as deficiências encontradas devem ser efetivamente gerenciadas por estes ${ }^{(12,22)}$.

Nesse contexto, espera-se que este trabalho forneça aos gestores uma avaliação que subsidie o planejamento de ações que retifiquem as inadequações encontradas nas unidades de saúde e permita uma visão da operacionalidade da $A B$, das facilidades e dos nós críticos que afetam a boa qualidade da atenção. Entretanto, este estudo possui limitações. Trata-se de uma pesquisa com delineamento transversal circunscrita ao cenário das unidades da ESF de um município. Não foram utilizadas técnicas de observação direta, mas, sim, analisados dados oriundos da autoavaliação das equipes de saúde da família.

\section{CONCLUSÃO}

A qualidade da infraestrutura das unidades de saúde e dos equipamentos para ações na $A B$ obteve uma avaliação predominantemente negativa pelas equipes de saúde da família. Evidenciaram-se fragilidades que requerem maior atenção: disponibilidade de linha telefônica e equipamentos de informática com acesso à internet, veículo oficial para deslocamento; materiais e equipamentos para a assistência inicial nas situações de urgência e emergência; e inadequação das unidades para atender portadores de deficiência, analfabetos e idosos.

A infraestrutura frágil e a insuficiência de recursos materiais foram uma realidade constatada no cenário avaliado, também verificada nos cuidados primários de saúde no Brasil. As inadequações das unidades de saúde prejudicam o desenvolvimento das ações na $A B$, a qualidade do cuidado e geram insatisfação nos profissionais e nos usuários. E limitam o potencial dos serviços para a consolidação da ESF, na perspectiva da reorganização de práticas centradas na tríade indivíduo-família-comunidade e na qualidade da atenção à saúde.

\section{- REFERÊNCIAS}


1. Ministério da Saúde (BR). Secretaria de Atenção à Saúde. Departamento de Atenção Básica. AMAQ: autoavaliação para melhoria do acesso e da qualidade. Brasília: Ministério da Saúde; 2013.

2. Felisberto DF, Silva GS, Beirão ROS, Falcão MLP, Felisberto E, de Albuquerque AC. Qualidade dos serviços oferecidos em uma unidade de saúde da família: avaliando a conformidade com os padrões do Programa Avaliação para Melhoria da Qualidade. Rev Baiana Saúde Pública. [Internet] 2013;37(2) [acesso em 12 fev 2016]. Disponível: http://inseer.ibict.br/rbsp/index.php/rbsp/article/viewFile/384/pdf_420.

3. Lins CFM, Alchieri JC, de Araújo Neto JL, Melo FAF. Avaliação da Estratégia Saúde da Família em Natal a partir das crenças dos seus colaboradores. Psico. [Internet] 2013;44(4) [acesso em 10 fev 2016]. Disponível: http:// revistaseletronicas.pucrs.br/ojs/index.php/revistapsico/article/view/12193/10846.

4. Cardoso AVL, Chain APN, Mendes RIP, Ferreira e Ferreira E, Vargas AMD, Martins AMEBL, et al. Assessment of the management of the Family Health Strategy via the tool Assessment for Quality Improvement in municipalities of Minas Gerais, Brazil. Ciênc. saúde coletiva. [Internet] 2015;20(4) [acesso em 12 fev 2016]. Disponível: http:// dx.doi.org/10.1590/1413-81232015204.01832014.

5. de Oliveira Junior RG, Santos EO, Ferraz CAA, de Lavor EM, Nunes LMN. Condições de trabalho das Equipes de Saúde da Família do município de Petrolina-PE: percepção dos profissionais de saúde. Mundo Saúde. [Internet] 2013;37(4) [acesso em 10 fev 2016]. Disponível: http://www.saocamilo-sp.br/pdf/mundo_saude/155558/A08.pdf.

6. Souza ROA, Machado CV, Noronha MF. Desafios da gestão municipal da atenção básica em saúde no brasil: um estudo de caso. Rev APS. [Internet] 2015;18(2) [acesso em 11 fev 2016]. Disponível: https://aps.ufjf.emnuvens. com.br/aps/article/view/2476/875.

7. Starfield B. Atenção Primária: equilíbrio entre necessidades de saúde, serviços e tecnologia. Brasília: Unesco/ Ministério da Saúde; 2002.

8. Rocha ACD, Sousa CPC, Queiroz D, Pedraza DF. Atenção básica à saúde: avaliação de estrutura e processo. RAS. 2012;14(54):71-9.

9. Kringos DS, Boerma W, van der Zee J, Groenewegen P. Europe's strong primary care systems are linked to better population health but also to higher health spending. Health Aff. [Internet] 2013;32(4) [acesso em 12 fev 2016]. Disponível: http://dx.doi.org/10.1377/hlthaff.2012.1242.

10. Soares Neto JJ, Machado MH, Alves CB. The Mais Médicos (More Doctors) Program, the infrastructure of primary health units and the municipal human development index. Ciênc. saúde coletiva. [Internet] 2016;21(9) [acesso em 22 nov 2016]. Disponível: http://dx.doi.org/10.1590/1413-81232015219.16432016.

11. Instituto Brasileiro de Geografia e Estatística (IBGE) Cidades@ Minas Gerais Montes Claros. [Internet] Rio de Janeiro: IBGE; 2010 [acesso em 22 fev 2016]. Disponível: http://cidades.ibge.gov.br/xtras/perfil. php?codmun=314330.

12. Magnago C, Pierantoni CR. Dificuldades e estratégias de enfrentamento referentes à gestão do trabalho na Estratégia Saúde da Família, na perspectiva dos gestores locais: a experiência dos municípios do Rio de Janeiro (RJ) e Duque de Caxias (RJ). Saúde debate. [Internet] 2015;39(104) [acesso em 12 fev 2016]. Disponível: http:// dx.doi.org/10.1590/0103-110420151040194.

13. Pimentel FC, de Albuquerque PC, de Souza WV. A Estratégia Saúde da Família no estado de Pernambuco: avaliação da estrutura das equipes por porte populacional. Saúde debate. [Internet] 2015;39(104) [acesso em 12 fev 2016]. Disponível: http://dx.doi.org/10.1590/0103-110420151040138.

14. Martins KP, da Costa TF, de Medeiros TM, Fernandes MGM, de França ISX, Costa KNFM. Internal structure of Family Health Units: access for people with disabilities. Ciênc. saúde coletiva. [Internet] 2016;21(10) [acesso em 22 nov 2016]. Disponível: http://dx.doi.org/10.1590/1413-812320152110.20052016.

15. Nora CRD, Junges JR. Humanization policy in primary health care: a systematic review. Rev. Saúde Pública. [Internet] 2013;47(6) [acesso em 12 fev 2016]. Disponível: http://dx.doi.org/10.1590/S0034-8910.2013047004581.

16. Tomasi E, Oliveira TF, Fernandes PAA, Thumé E, da Silveira DS, Siqueira FV, et al. Estrutura e processo de trabalho na prevenção do câncer de colo de útero na Atenção Básica à Saúde no Brasil: Programa de Melhoria do Acesso e da Qualidade - PMAQ. Rev. Bras. Saude Mater. Infant. [Internet] 2015;15(2) [acesso em 12 fev 2016]. Disponível: http://dx.doi.org/10.1590/S1519-38292015000200003. 
17. Oliveira DC, Mandú ENT, Corrêa ACP, Tomiyoshi JT, Teixeira RC. Estrutura organizacional da atenção pósparto na Estratégia Saúde da Família. Esc. Anna Nery. [Internet] 2013;17(3) [acesso em 12 fev 2016]. Disponível: http://dx.doi.org/10.1590/S1414-81452013000300007.

18. Turci MA, Lima-Costa MF, Macinko J. Influência de fatores estruturais e organizacionais no desempenho da atenção primária à saúde em Belo Horizonte, Minas Gerais, Brasil, na avaliação de gestores e enfermeiros. Cad. Saúde Pública. [Internet] 2015;31(9) [acesso em 12 fev 2016]. Disponível: http://dx.doi.org/10.1590/0102311X00132114.

19. Mudrick NR, Breslin ML, Liang M, Yee S. Physical accessibility in primary health care settings: results from California on-site reviews. Disabil Health J. [Internet] 2012;5(3) [acesso em 10 fev 2016]. Disponível: http://dx.doi. org/10.1016/j.dhjo.2012.02.002.

20. Gudlavalleti MVS, John N, Allagh K, Sagar J, Kamal-akannan S, Ramachandra SS. Access to health care and employment status of people with disabilities in South India, the SIDE (South India Disability Evidence) study. BMC Public Health. [Internet] 2014;(14) [acesso em 10 fev 2016]. Disponível: http://dx.doi.org/10.1186/1471-245814-1125.

21. Lagu T, lezzoni LI, Lindenauer PK. The axes of access - improving care for patients with disabilities. N Engl J Med. [Internet] 2014;370(19) [acesso em 10 fev 2016]. Disponível: http://dx.doi.org/10.1056/NEJMsb1315940.

22. de Mesquita KO, da Silva LCC, Lira RCM, Freitas CASL, Lira GV. Patient safety in primary health care: an integrative review. Cogitare Enferm. [Internet] 2016;21(2) [acesso em 22 nov 2016]. Disponível: http://dx.doi. org/10.5380/ce.v21i2.45665. 